\title{
Tropical Plucker functions and Kashiwara crystals
}

\author{
V.I. Danilov, A.V. Karzanov and G.A. Koshevoy
}

\begin{abstract}
We show that Kashiwara crystals of type $A$ can be described by use of tropical Plucker functions (TP-functions) on a hyper-cube and related rhombus tilings. We also illustrate the formation of crystals of types $B$ and $C$ via symmetric TP-functions and tilings.
\end{abstract}

\section{Introduction}

The notion of crystal introduced by Kashiwara [KN-94] has proved its importance in representation theory. A finite crystal is a finite edge-colored digraph (directed graph) in which each connected monochromatic subgraph is a simple path and there are certain interrelations on the lengths of such paths, described via coefficients of an Cartan matrix (features of this matrix characterize the crystal type). There are several models to characterize crystals for a variety of types; e.g., via generalized Young tableaux [KN-94], Littelmanns path model [Lit-95], MV-polytopes [Kam-10], the crossing model [DKK-08].

In this paper we propose a new model for $n$-colored crystals of type $A$ : the vertices of the free crystal graph are identified with integer-valued tropical Plücker functions (briefly, TP-functions) on the $n$-dimensional Boolean cube and the edges of color $i$ (yielding the crystal operation $\mathbf{i}$ ), $i=1, \ldots, n$, are defined by use of the restriction of TP-functions to a rhombus tiling which is adapted (in a certain sense) to this color. The subgraph whose vertices correspond to the submodular TP-functions is isomorphic to the crystal graph $B(\infty)$ which serves a 'combinatorial skeleton' of the canonical basis introduced by Lusztig [Lu-93]. It is well-known that a submodular function on a Boolean cube defines a polymatroid. If a submodular function is additionally a TP-function, then the corresponding polymatroid is an MV-polytope of type A.

This model of $A$-type crystals is symmetric with respect to the inversion of colors. This allows us to get a transparent construction of crystals of types $B$ and $C$ by considering the corresponding symmetric TP-functions and rhombus tilings. Here we rely on a classical technique of 'folding' for Dynkin diagrams and the result that $B$ - and $C$-crystals can be produced from the inversion-invariant parts of symmetric $A$-crystals, see, e.g., [NS-01] (a direct combinatorial proof of that result

2010 Mathematics Subject Classification. 05E10, 20G42,52C20.

Key words and phrases. Crystals, Plücker relations, tiling diagrams. 
is given in [DKK-12], based on the crossing model for $A$-crystals [DKK-08] and the so-called worm model for 2-colored $B$-crystals [DKK-09]).

Working with the TP-model of an $A$-crystal, we also give a transparent description of those vertices (TP-functions) that form the so-called principal lattice of the crystal. (The existence of this object possessing a number of nice structural properties was revealed in [DKK-08].)

The structure of this paper is as follows. In Section 2 we recall definitions and some basic facts on tropical Plücker functions and rhombus tilings. Section 3 introduces crystal actions (operators) on the set of TP-functions, forming an edgecolored digraph, and shows that this digraph is indeed the free crystal of type $A$. Then we explicitly distinguish in it the subgraph which is isomorphic to the crystal $B(\infty)$ (for the given type $A$ ). Section 4 focuses on finite subcrystals of the constructed free (or $B(\infty)$ ) crystal, which are typically of more interest for purposes of representation theory. Section 5 is devoted to a description of crystals of types $B$ and $C$ by use of symmetric TP-functions on an $n$-cube (where type $B$ (resp. $C$ ) takes place when $n$ is odd (resp. even)). Sections 6 and 7 contain proofs of two theorems stated in Section 4.

\section{Tropical Plucker functions}

2.1. For a positive integer $n$, the set $\{1, \ldots, n\}$ equipped with the natural order $1<2<\ldots<n$ is denoted by $[n]$. The collection of all subsets in $[n]$ forms the Boolean $n$-cube $2^{[n]}$. For $A \subseteq[n]$, we write $|A|$ for the cardinality of $A$.

Definition. A function $f: 2^{[n]} \rightarrow \mathbb{R}$ is called a tropical Plücker function (or a $T P$-function for short) if for any subset $A \subset[n]$ and any three elements $i<j<k$ in $[n]-A$, the following $T P$-relation hold:

$$
f(A j)+f(A i k)=\max (f(A i)+f(A j k), f(A k)+f(A i j)) .
$$

Hereinafter for brevity we write $A i^{\prime} \ldots i^{\prime \prime}$ for $A \cup\left\{i^{\prime}, \ldots, i^{\prime \prime}\right\}$. The set of integervalued TP-functions is denoted by $T P_{n}$, whereas the notation $T P_{n}(\mathbb{R})$ will be used for real-valued TP-functions.

2.2. As a subset of the space $\mathbb{R}^{[n]}$ of all function on $[n]$, the set $T P_{n}(\mathbb{R})$ preserves under multiplication by positive factors, but does not under addition. More precisely, $T P_{n}(\mathbb{R})$ is a (nonconvex) cone having lineal of dimension $2 n$ formed by the so-called principal TP-functions; so addition (or subtraction) of a principal TP-function to (from) any TP-function makes a TP-function as well. Principal TP-functions are: (i) any affine function of the form $\alpha+\mu(A)$, where $\alpha \in \mathbb{R}$ and $\mu$ is an additive measure on $2^{[n]}$, and (ii) any function $f(A)$ depending on the cardinality $|A|$ of a subset $A \subseteq[n]$. The sum of functions of these two types is again a principal TP-function, and moreover, one shows that every principal TP-function is obtained in this way. This implies that the space of principal TP-functions has dimension just $2 n$.

2.3. TP-functions can be freely defined on certain collections of subsets in $[n]$. More precisely, a collection (set-system) $\mathcal{B} \subseteq 2^{[n]}$ is called a TP-basis, or simply a basis, if each TP-function is determined by its values on $\mathcal{B}$, and moreover, the values on $\mathcal{B}$ can be chosen arbitrarily. Such bases do exist, and TP-bases of our 
especial interest will be those related to rhombus tilings on a zonogon. Let us briefly recall these notions, referring to [DKK-09b] for details. (For a wider discussion on TP-functions, TP-bases, rhombus tilings, and related topics, see also [DKK-10, DKK-10b].)

Rhombus tiling diagrams live within a zonogon; the latter is defined as follows. In the upper half-plane, take $n$ vectors $\xi_{1}=\left(a_{1}, 1\right), \xi_{2}=\left(a_{2}, 1\right), \ldots, \xi_{n}=\left(a_{n}, 1\right)$ so that $a_{1}<a_{2}<\ldots<a_{n}$. Then the set $Z=Z_{n}:=\left\{\lambda_{1} \xi_{1}+\ldots+\lambda_{n} \xi_{n}: 0 \leq\right.$ $\left.\lambda_{i} \leq 1, i=1, \ldots, n\right\}$ is a $2 n$-gone. Moreover, $Z$ is a zonogon, as it is the sum of $n$ line-segments $\left\{\lambda \xi_{i}: 1 \leq \lambda \leq 1\right\}, i=1, \ldots, n$. Also $Z$ is the image by the linear projection $\pi$ of the solid cube $[0,1]^{[n]}$ into the plane, defined by $\pi(x):=$ $x_{1} \xi_{1}+\ldots+x_{n} \xi_{n}$. The boundary $b d(Z)$ of $Z$ consists of two parts: the left boundary, $l b d(Z)$, formed by the points (vertices) $z_{i}^{\ell}:=\xi_{1}+\ldots+\xi_{i}(i=0, \ldots, n)$ connected by the line-segments $z_{i-1}^{\ell} z_{i}^{\ell}:=z_{i-1}^{\ell}+\left\{\lambda \xi_{i}: 0 \leq \lambda \leq 1\right\}$, and the right boundary, $\operatorname{rbd}(Z)$, formed by the points $z_{i}^{r}:=\xi_{i+1}+\ldots+\xi_{n}(i=0, \ldots, n)$ connected by the line-segments $z_{i}^{r} z_{i-1}^{r}$. So $z_{0}^{\ell}=z_{n}^{r}$ is the minimal vertex of $Z$ and $z_{n}^{\ell}=z_{0}^{r}$ is the maximal vertex.

A subset $X \subseteq[n]$ is identified with the corresponding vertex of the $n$-cube and with the point $\sum_{i \in X} \xi_{i}$ in the zonogon $Z$. Due to the above independence condition, all such points in $Z$ are different.

By a tile we mean a parallelogram $R$ of the form $X+\left\{\lambda \xi_{i}+\lambda^{\prime} \xi_{j}: 0 \leq \lambda, \lambda^{\prime} \leq 1\right\}$, where $X \subset[n]$ and $1 \leq i<j \leq n$; we also say that $R$ is the $i j$-tile at $X$ and denote it by $R(X ; i, j)$. (Sometimes we may conditionally call $R$ a rhombus, as the choice of base vectors $\xi_{1}, \ldots, \xi_{n}$ that have equal lengths would not affect our description in essence.) According to a natural visualization of $R$, its vertices $X, X i, X j, X i j$ are called the bottom, left, right, top vertices of $R$ and denoted by $b(R), \ell(R), r(R)$, $t(R)$, respectively. We say that a point (subset) $Y \subseteq[n]$ has height $|Y|$.

2.4. Definition. A (rhombus) tiling diagram, or a tiling for short, is a collection $T$ of tiles which subdivide the zonogon $Z=Z_{n}$ (i.e., the tiles cover $Z$ and their interiors are disjoint).

Here are two possible tilings of the zonogon (hexagon) $Z_{3}$.
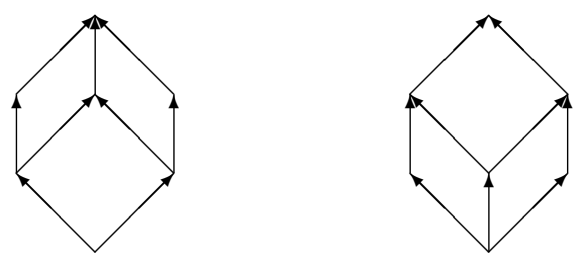

The set of vertices (edges) of a tiling $T$ is the union of vertices (resp. edges) of tiles in $T$. We direct all edges upward and say that an edge parallel to $\xi_{i}$ has color $i$. The set of vertices of $T$ gives a collection of subsets of $[n]$, called the spectrum of $T$ and denoted by $S p(T)$. Note that the boundary vertices of $Z_{n}$, viz. the sets $\emptyset,\{1\},\{1,2\}, \ldots,\{1,2, \ldots, n\},\{2, \ldots, n\}, \ldots,\{n\}$, belong to the spectrum of every tiling.

2.5. Example: the standard tiling. An interval in $[n]$ is a subset of the form $\{i, i+1, \ldots, j\}$; the empty set $\emptyset$ is regarded as an interval as well. The standard tiling is the tiling $T$ whose spectrum consists of all intervals. Any tile $R$ of $T$ is 
viewed as follows: if the bottom vertex $b(R)$ is an interval $I=\{i+1, \ldots, j-1\}$, then the left, right, and top vertices are the intervals $l(R)=I i, r(R)=I j$, and $t(R)=I i j$, respectively. The standard tiling for $n=6$ is drawn in the picture.

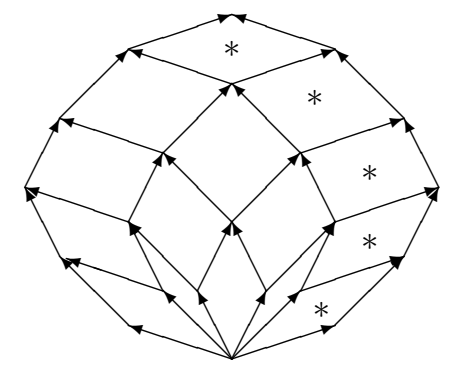

2.6. It is shown in $[\mathbf{D K K} \mathbf{- 0 9 b}]$ that for every tiling $T$, the restriction map

$$
T P_{n}(\mathbb{R}) \rightarrow \mathbb{R}^{S p(T)}
$$

is a bijection (and similarly when $\mathbb{R}$ is replaced by $\mathbb{Z}$ ). This map is piecewise linear.

One can think of the tilings as playing the role of 'charts' for $T P_{n}$. It is important to understand how the corresponding 'coordinates' are modified when one chart is replaced by another. The simplest case is when two tilings $T$ and $T^{\prime}$ are connected by a flip. More precisely, suppose that $T$ contains a hexagon $H$, i.e. the image by $\pi$ of some 3 -dimensional face of the solid $n$-cube. Then $H$ is subdivided into three tiles in $T$ (see the illustration in Section 2.4). Changing these three tiles by the other possible combination of three tiles (and keeping all the tiles outside $H$ ), we obtain a new tiling $T^{\prime}$. We say that these $T$ and $T^{\prime}$ are connected by one flip. All vertices of $T$ and $T^{\prime}$ are the same except for two vertices, which are of the form $A j$ and $A i k$ (for some $A$ and $i<j<k$ ) and are interchanged under the flip. Recall that the values $f(A j)$ and $f(A i k)$ are linked by the TP-relation (2.1).

An important fact shown in [HS-07] is that any two tilings are connected by a sequence of flips.

2.7. It is often more convenient to work with the excesses of a TP-function on tiles of a tiling rather than the values of the function themselves.

Let $R$ be an $i j$-tile with bottom vertex $A$. For a function $f$ on $2^{[n]}$, the excess of $f$ on $R$ is defined to be the number

$$
\varepsilon(f, R):=f(A i)+f(A j)-f(A)-f(A i j) .
$$

For a tiling $T$, if the values of a TP-function $f$ on the vertices of the right boundary of the zonogon are fixed, then the excesses of $f$ on the tiles of $T$ determine the values of $f$ on all vertices of $T$.

To see this, let us use the notion of a snake in a tiling $T$. This is a directed path $P$ in $T$ (considered as digraph) which goes from the bottom vertex $\emptyset$ to the top vertex $[n]$ of the zonogon $Z$. The length (number of edges) of $P$ is equal to $n$, and $k$-th edge of $P$ is congruent to some base vector $\xi_{i_{k}}$. Thus, the snake gives the sequence $\left(i_{1}, \ldots, i_{n}\right)$ of elements of $[n]$. It is easy to show that all numbers $i_{k}$ in this sequence are different; this property allows us to identify a snake with the 
corresponding permutation of the set $[n]$ (or with a complete flag of subsets in $[n]$ ). We use the following simple fact.

Lemma (see [El-97, Lemma 2.1]). If a snake of $T$ is different from the left boundary $l b d(Z)$ of $Z$, then there exists a tile $R$ of $T$ such that both right edges of $R$ belong to the snake.

Now the above assertion is obtained as follows. Assume that the values of $f$ are already known for all vertices of $T$ lying on the right from some snake $P \neq l b d(Z)$ (including $P$ ). Take a tile $R$ as in the lemma. Since the values of $f$ at the three vertices $b(R), r(R), t(R)$ of $R$ are known, we can compute the value at the fourth (left) vertex $l(R)$ using the excess of $f$ on $R$. Accordingly, update $P$ by replacing the right edges of $R$ by the left edges in it. Continue the process until we get $l b d(Z)$.

2.8. In the previous section we introduced the notion of a snake in a tiling. For further purposes, it is useful to define an abstract snake to be an arbitrary complete flag $S_{\bullet}$ of subsets of $[n]$ :

$$
S_{0}=\emptyset \subset S_{1} \subset \ldots \subset S_{n}=[n], \quad\left|S_{k}\right|=k .
$$

Such a flag determines a path in the zonogon from the bottom to the top. This path (which we call an abstract snake as well) divides the zonogon into two parts the left and right ones. For each part, one can speak about rhombus tilings in it. A basic fact about these tilings (see [DKK-09b]) is that for each part $Z^{\prime}$, at least one tiling in $Z^{\prime}$ exists (unless the snake coincides with the left or right boundary of the zonogon) and any two tilings in $Z^{\prime}$ are connected by a chain of flips. In particular, any abstract snake is realized as a snake of some tiling of the whole zonogon.

2.9. There are many interrelations between the excesses on different tiles. We restrict ourselves by exposing such interrelations for the key case $n=3$. The picture below illustrates two tilings of the hexagon $Z_{3}$ and labels the excesses on six tiles.
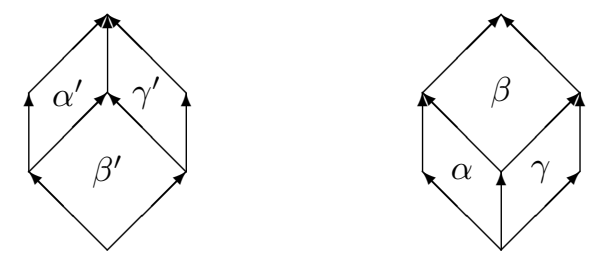

It is easy to check that these excesses satisfy the linear relations

$$
\begin{aligned}
& \alpha+\beta=\beta^{\prime}+\gamma^{\prime}, \\
& \alpha^{\prime}+\beta^{\prime}=\beta+\gamma .
\end{aligned}
$$

Adding up these relations, we obtain one more identity

$$
\alpha+\alpha^{\prime}=\gamma+\gamma^{\prime} .
$$

If $f$ is a TP-function, we also should add two inequalities

$$
\begin{gathered}
\beta=\min \left(\alpha^{\prime}, \gamma^{\prime}\right), \\
\beta^{\prime}=\min (\alpha, \gamma) .
\end{gathered}
$$


We can take arbitrary numbers $\alpha, \beta, \gamma$ and define $\alpha^{\prime}, \beta^{\prime}, \gamma^{\prime}$ with help of the above relations (and vice versa). A 'more symmetric' way is to begin with the quadruple $\alpha, \gamma, \alpha^{\prime}, \gamma^{\prime}$ satisfying (2.5) and express $\beta$ and $\beta^{\prime}$ by using formulas (2.6) and (2.7). The 'most exotic' way is to deal with the quadruple $\beta, \gamma, \beta^{\prime}, \gamma^{\prime}$ satisfying two inequalities $\beta \leq \gamma^{\prime}$ and $\beta^{\prime} \leq \gamma$ and the condition that at least one of them holds with equality. Then $\alpha$ is expressed as $\beta^{\prime}+\gamma^{\prime}-\beta$, whence $\alpha \geq \beta^{\prime}$. Since $\gamma \geq \beta^{\prime}$ and since $\beta \leq \gamma^{\prime}$ or $\beta^{\prime} \leq \gamma$ turns into equality, we obtain $\beta^{\prime}=\min (\alpha, \gamma)$. Similarly for $\alpha^{\prime}$.

As a consequence, we conclude that if $\alpha, \beta, \gamma$ are nonnegative, then $\alpha^{\prime}, \beta^{\prime}, \gamma^{\prime}$ are nonnegative as well, and vice versa. Moreover, if $\alpha, \alpha^{\prime}=0$, then $\beta, \gamma, \beta^{\prime}, \gamma^{\prime}=0$.

2.10. Here we give an excess description of principal TP-functions.

Proposition. Let $f$ be a TP-function. The following assertions are equivalent:

(i) the function $f$ is a principal TP-function;

(ii) if $R$ and $R^{\prime}$ are two tile having the same height, then $\varepsilon(f, R)=\varepsilon\left(f, R^{\prime}\right)$;

(iii) the same as in (ii) when both $R, R^{\prime}$ belong to the same tiling of the zonogon.

Proof. Let $f$ be a principal TP-function, i.e. $f$ is the sum of an affine function and a cardinality function of the form $\psi(|\cdot|)$ (see 2.2). Since affine functions have zero excesses (and are characterized by this property), one may assume that $f(\cdot)=\psi(|\cdot|)$. Then the excess on a tile depends only on the height of the tile (and is equal to the 'second difference' of the function $\psi$ ). This proves the implication (i) $\Rightarrow$ (ii). The implication (ii) $\Rightarrow$ (iii) is trivial.

Now suppose that (iii) is valid. Subtracting from $f$ an appropriate function $\psi(|\cdot|)$, we may assume that all tiles of a tiling $T$ have zero excesses. Subtracting an appropriate affine function, we may assume that $f$ has zero values on the right boundary of the zonogon. Then (see 2.7) $f$ is 0 at every vertex of $T$. According to 2.6 , we obtain that $f$ is 0 everywhere.

\section{The structure of $A_{n}$-crystals on TP-functions}

3.1. A pre-crystal (with $n$ colors) is a certain digraph $K=(V(K), E(K))$ in which each edge is colored by one of colors $1, \ldots, n$. In other words, the edge set $E(K)$ is partitioned into $n$ subsets $E_{1}, \ldots, E_{n}$; we say that an edge in $E_{i}$ has color $i$. The first axiom of pre-crystals says that for any color $i$, the subgraph $\left(V(K), E_{i}\right)$ is a disjoint union of (simple directed) paths which may be finite or infinite. The move along an edge $e=(u, v)$ of color $i$ is understood as action of (crystal) operator $\mathbf{i}$ at the vertex $u$, and we write $\mathbf{i} u=v$. This is a partial operator on $V(K)$, and if there is no edge of color $i$ leaving a vertex $u$, then we say that $\mathbf{i}$ does not act at $u$.

The inverse (partial) operator $\mathbf{i}^{-1}$ is defined in a natural way: if $\mathbf{i}$ acts at $u$ and $v=\mathbf{i} u$, then $\mathbf{i}^{-1}$ acts at $v$ and $\mathbf{i}^{-1} v=u$.

A morphism $K \rightarrow K^{\prime}$ of two $n$-colored pre-crystals is a mapping $\varphi: V(K) \rightarrow$ $V\left(K^{\prime}\right)$ which commutes with the operators $\mathbf{i}, i=1, \ldots, n$, in the sense that if $\mathbf{i}$ acts at a vertex $v$ of $K$, then $\mathbf{i}$ acts at the vertex $\varphi(v)$ of $K^{\prime}$, and $\varphi(\mathbf{i} v)=\mathbf{i} \varphi(v)$.

3.2. The second axiom of pre-crystals involves a (generalized) Cartan matrix $M=$ $\left(m_{i j}\right)$, where $i, j \in[n]$. The entries of $M$ are integers satisfying $m_{i i}=2$ and $m_{i j} \leq 0$ for $i \neq j$. We denote $i$-th row $\left(m_{i 1}, \ldots, m_{i n}\right)$ of $M$ by $m_{i}$, and consider the rows as elements of the abelian group $\mathbb{Z}^{n}$. 
When $m_{i j}$ is -1 for $|i-j|=1$, and 0 for the other $i \neq j$, the (pre-)crystals related to $M$ are said to have type $A$ or, more precisely, type $A_{n}$.

The (full) commutative $M$-pre-crystal is meant to be the set $\mathbb{Z}^{n}$ in which the action of each crystal operator $\mathbf{i}$ consists in adding the vector $m_{i}$. A pre-crystal $K$ is called an $M$-pre-crystal if there exists a morphism, also called a weight mapping, of $K$ to the commutative $M$-pre-crystal.

However, just non-commutative (pre-)crystals are of most interest in representation theory. In particular, so are Kashiwara crystals (associated with irreducible highest weight integrable modules over quantized universal enveloping algebras). In what follows we restrict ourselves by the case of a non-degenerate Cartan matrix (with linearly independent row vectors $m_{i}$ ). For elements (vertices) $u, v$ of a pre-crystal $K$, we write $u \preceq v$ if there exists a word $\mathbf{w}$ using letters $\mathbf{i}$ (but not their inverse ones), $i=1, \ldots, n$, such that $v=\mathbf{w} u$. In other words, in the digraph $K$ there is a directed path from $u$ to $v$. The binary relation $\preceq$ is reflexive and transitive. The existence of a weight mapping together with linear independence of $m_{i}$ 's implies antisymmetry of $\preceq$. Thus, $\preceq$ is a partial order on the set of vertices of $K$. Moreover, the poset $(V(K), \prec)$ is $\mathbb{Z}^{n}$-graded, i.e., for each $i$, any closed (nondirected) cycle in $K$ has equal numbers of forward and backward edges of color $i$.

When $K$ has a (unique) vertex $v$ such that $v \preceq w$ (resp. $v \succeq w$ ) for all vertices $w$, we call $v$ the source (resp. sink) of $K$. Relying on [KN-94], we say that an $M$-pre-crystal $K$ having source (or sink) is an $M$-crystal if for any two colors $i, j$, the subgraph of $K$ induced by the edges of colors $i$ and $j$ yields an $M[i, j]$-crystal. In other words, in order to define $n$-colored crystals, it suffices to be able to define two-colored ones. An $M$-pre-crystal without source and sink is a free $M$-crystal if, for any $M$-crystal $K$, there is a morphism of $K$ into the free $M$-crystal. For types $A, B, C$, there have been known nice local axiomatics and direct combinatorial constructions; see [Ste-03] (for local axioms of simply-laced crystals), [DKK-07] (for $A_{2}$ ), and [DKK-09] (for $B_{2}$ ); in its turn, type $C_{2}$ is obtained from $B_{2}$ by swapping the colors.

3.3. We will use some facts about $A_{n}$-crystals from [DKK-08]. Let $K$ be an $A_{n^{-}}$ crystal with source $s$. Let $c_{i}$ denote the maximal length of a path of color $i$ beginning at $s$. This is a nonnegative integer or $\infty$. It turns out that the tuple $c=\left(c_{1}, \ldots, c_{n}\right)$ of these numbers (parameters of the crystal) determines the crystal. Moreover, for any $c$, there exists a (unique) crystal with these parameters $c$; we denote it by $K(c)$. If all numbers $c_{i}$ are finite, then the crystal $K(c)$ is finite and has sink. Reversing the colors (i.e., replacing $i$ by $n+1-i$ ), we obtain the crystal $K(\bar{c})$, where $\bar{c}_{i}:=c_{n+1-i}$. And reversing the edges of $K(c)$ while preserving their colors, we again obtain the crystal (isomorphic to) $K(\bar{c}$ ).

There is a unique morphism of $K(c)$ to the crystal $K_{0}:=K(+\infty, \ldots,+\infty)$ which sends the source of the first crystal to the source $s_{0}$ of the second one. The image in $K_{0}$ of the sink of $K(c)$ is called a principal vertex and denoted by $p(c)$. The set of vertices $p(c)$ (over all $c$ ) is called the principal lattice of $K_{0}$; as a poset, it is isomorphic to $\mathbb{Z}_{+}^{n}$ equipped with the coordinate-wise order. To obtain a finite crystal, one should take a principal vertex $p$ and form the interval between the source $s_{0}$ and $p$ in $K_{0}$ (i.e., the subgraph formed by the vertices and edges contained in paths from $s_{0}$ to $p$ ). 
3.4. Next we describe the structure of an $n$-colored pre-crystal (an $A_{n}$-crystal, in fact) on the set $T P=T P_{n+1}(\mathbb{Z})$ of integer TP-functions on $2^{[n+1]}$. We need to introduce two notions.

A tile $R$ on the zonogon $Z=Z_{n+1}$ is called left if both of its left edges belong to the left boundary of $Z$; in this case we also say that $R$ is pressed to $l b d(Z)$. If $R$ is situated at height $h$, then its bottom, left, and top vertices are $b(R)=[h-1]$, $l(R)=[h]$, and $t(R)=[h+1]$. We will denote such an $R$ by $L R_{h}$. The right tile $R R_{h}$ at height $h$ is defined in a similar way w.r.t. $\operatorname{rbd}(Z)$.

Let us say that a tiling $T$ of the zonogon $Z$ fits to a (crystal) color $i$ ( $i=$ $1, \ldots, n)$ if $T$ contains the left tile $L R_{i}$. By reasonings in 2.7, for any $i$, there exists a tiling which fits to color $i{ }^{1}$

Now we can define the action of operator $\mathbf{i}$ on a TP-function $f \in T P$. Choose a tiling $T$ fitting to color $i$. Then the function $\mathbf{i} f$ is defined by the rule

$$
(\mathbf{i} f)(v)= \begin{cases}f(v)+1, & \text { if } v=[i], \\ f(v) & \text { for the other vertices } v \text { of } T\end{cases}
$$

(taking into account that $S p(T)$ is a TP-basis; see 2.6). In other words, within the 'chart' $T$, the action of $\mathbf{i}$ is simply the increase by 1 of the value of $f$ at the only vertex $v=[i]$. Note that this action may cause changes of $f$ at many vertices of the Boolean cube which are beyond $S p(T)$. We have the following important property:

The operator $\mathbf{i}$ as in (3.1) is well-defined, that is it does not depend on the choice of a tiling fitting to color $i$.

This follows from the fact that any two tilings containing the tile $L R_{i}$ are connected by a sequence of flips which do not change the tile $L R_{i}$ (by reasonings in 2.8).

3.5. Theorem. The operators $\mathbf{i}(i=1, \ldots, n)$ endow the set $T P=T P_{n+1}$ with the structure of an $A_{n}$-crystal.

Proof. It is easy to see that each operator $\mathbf{i}$ is invertible (as we always can reduce by 1 the value at the vertex $[i]$ of a tiling fitting to color $i$ ). Therefore, each orbit produced by operator $\mathbf{i}$ is a path infinite in both directions. Assigning to a TP-function $f$ the vector $\sum_{i=1, \ldots, n} f([i]) a_{i} \in \mathbb{Z}^{n}$ (where $a_{i}$ is $i$-th row of the Cartan matrix for $A_{n}$ ), we obtain an appropriate weight morphism.

So we have an $A_{n}$-pre-crystal $K$ on TP and wish to show that it is $A_{n}$-crystal. To this aim, consider actions of two operators $\mathbf{i}$ and $\mathbf{j}$.

If $j \neq i \pm 1$, then $\mathbf{i}$ and $\mathbf{j}$ commute. Indeed, in this case there exists a tiling fitting simultaneously to both colors $i$ and $j$.

Now suppose that $j=i+1$. We have to show that the restriction to colors $i, j$ produces an $A_{2}$-crystal. Equivalently, we may assume that $n=3$. Moreover, one may consider only $T P_{3}$-functions $f$ that are equal to 0 at the right boundary of the hexagon $Z_{3}$ (see explanations in 3.7 below). We show that our pre-crystal $K$ is isomorphic to an $A_{2}$-crystal constructed by the crossing model from [DKK-07].

Let $a, b, c, c^{\prime}$ denote the values of a function $f$ as above at points $1,12,2,13$, respectively; see the picture.

\footnotetext{
${ }^{1}$ A tiling fitting to color $i$ corresponds to a reduced decomposition of the inverse permutation which starts with $i$-th transposition $s_{i}$.
} 


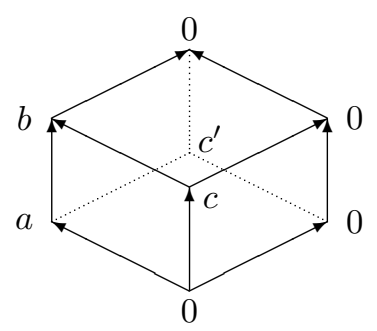

Since these values determine $f$, we can conclude that the vertices of $K$ one-to-one correspond to the quadruples $\left(a, b, c, c^{\prime}\right)$ satisfying the $T P_{3}$-relation

$$
c+c^{\prime}=\max (a+0, b+0)=\max (a, b) .
$$

The action of operator 1 consists in increasing $a$ by 1 , whereas $b$ and $c$ do not change. As to $c^{\prime}$, it increases by 1 if $a \geq b$, and preserves if $a<b$. Operator 2 acts in a similar way: it increases $b$ by 1 and increases $c$ if $b \geq a$, preserving the other values.

It is convenient to consider the excesses $\alpha, \beta, \gamma, \alpha^{\prime}, \beta^{\prime}, \gamma^{\prime}$ as in Section 2.9; in our case we have $\gamma=c, \gamma^{\prime}=c^{\prime}, \beta=b-c$, and $\beta^{\prime}=a-c^{\prime}$. Relation (3.2) is equivalent to the inequalities $\beta^{\prime} \leq c$ and $\beta \leq c^{\prime}$ together with the requirement that at least one of them turns into equality. In terms of excesses, operator $\mathbf{1}$ acts as follows: it increases $\beta^{\prime}$ by 1 if $\beta^{\prime}<\gamma$, and increases $\gamma^{\prime}$ by 1 if $\beta^{\prime}=\gamma$. Operator 2 acts in a symmetric way (updating $\beta, \gamma$ ). As a result, we obtain the behavior as described by the crossing model for $A_{2}$-crystals in [DKK-07].

This completes the proof of the theorem.

3.6. Proposition. The crystal operators $\mathbf{i}$ commute with the addition of any principal TP-function.

In other words, for any TP-function $f$ and any principal TP-function $p$,

$$
\mathbf{i}(f+p)=\mathbf{i} f+p .
$$

This follows from two observations. Firstly, if $+p$ is a TP-function. Secondly, this function coincides with the function $\mathbf{i}(f+p)$ within any tiling fitting to color $i$.

Thus, addition of a principal TP-function is an automorphism of the crystal $T P$.

3.7. Since the crystal operators do not change values of TP-functions on the right boundary of the zonogon $Z=Z_{n+1}$, these values give $n+2$ invariants for crystal actions (since $\operatorname{rbd}(Z)$ has $n+2$ vertices). This implies that the crystal $T P$ is not connected as graph. Fixing values $x$ on $\operatorname{rbd}(Z)$ (where $x$ is an $(n+2)$-vector), we obtain a subcrystal of $T P$, denoted as $K[x]$. We will show later that $K[x]$ is connected (see Corollary 4.5). Since the crystals $K[x]$ are isomorphic for all $x$ (which follows from Proposition 3.6), we can restrict ourselves by considering the crystal $K=K[0]$. Its vertex set consists of all integer TP-functions which take zero values on the right boundary of $Z$.

3.8. Consider the set $P$ of principal TP-functions belonging to the crystal $K=$ $K[0]$. It consists of principal TP-functions which are zero on the right boundary of the zonogon. By Proposition 2.10, such a function is determined by (arbitrarily) choosing excesses at heights $h=1, \ldots, n$. This gives a natural isomorphism between 
$P$ and $\mathbb{Z}^{n}$. Following $[\mathbf{D K K} \mathbf{- 0 8}]$, we call $P$ the principal lattice of the $A_{n}$-crystal $K$ (cf. 3.3). In Section 4 we will use the basis of the abelian group $P$ consisting of principal TP-functions $\pi_{1}, \ldots, \pi_{n}$, where $\pi_{i}$ is defined by the following three conditions:

a) $\pi_{i}$ takes zero values on the vertices of the right boundary of the zonogon;

b) $\pi_{i}$ has zero excess on any tile at height different from $n+1-i$;

c) $\pi_{i}$ has excess 1 on any tile at height $n+1-i$.

\section{Subcrystals in $K$}

The $A_{n}$-crystal $K$ formed in the previous section is not bounded (or free) in both forward and backward directions, the operators $\mathbf{i}$ and $\mathbf{i}^{-1}$ are defined everywhere, and any string (word) of such operators is applicable at any vertex. In this section we show that every finite $A$-crystal is realized as a subcrystal of this free crystal $K$.

We begin with a description of the order $\preceq$ on the group $P$ of principal elements of the crystal $K$. As is said in 3.8, this group is naturally identified with the group $\mathbb{Z}^{n}$.

4.1. Proposition. Under the identification of $P$ with $\mathbb{Z}^{n}$, the restriction of the order $\preceq($ on $V(K))$ to $P$ coincides with the coordinate-wise order on $\mathbb{Z}^{n}$.

We will prove this proposition in Section 4.5. Assuming its validity, we observe that the basic principal TP-functions $\pi_{i}$ (defined in 3.8) can be obtained from zero function by applying some words using 'letters' $\mathbf{1}, \mathbf{2}, \ldots \mathbf{n}$. Words of this sort are explicitly indicated in [DKK-08, Expression (6.4)], called fundamental words there, and we recall them to make our description more self-contained. Namely, we define (fundamental) words $\mathbf{W}_{1}, \mathbf{W}_{2}, \ldots, \mathbf{W}_{n}$ by

$$
\mathbf{W}_{i}=((\mathbf{n}-\mathbf{i}+\mathbf{1})(\mathbf{n}-\mathbf{i}+\mathbf{2}) \cdots \mathbf{n}) \cdot \ldots \cdot(\mathbf{2 3} \cdots(\mathbf{i}+\mathbf{1})) \cdot(\mathbf{1 2} \cdots \mathbf{i}) .
$$

Here $\mathbf{W}_{i}$ consists of $n-i+1$ 'blocks', and $j$-th 'block' (from right), where $j=$ $1, \ldots, n-i+1$, is the sequence of operators $\mathbf{j}, \ldots, \mathbf{j}+\mathbf{i}-\mathbf{1}$ (applied in the reverse order). Thus,

$$
\begin{gathered}
\mathbf{W}_{1}=(\mathbf{n}) \cdot \ldots \cdot(\mathbf{2}) \cdot(\mathbf{1}), \\
\mathbf{W}_{2}=((\mathbf{n}-\mathbf{1}) \mathbf{n}) \cdot \ldots \cdot(\mathbf{2 3}) \cdot(\mathbf{1 2}), \\
\ldots \\
\mathbf{W}_{n}=\mathbf{1 2} \cdots \mathbf{n} .
\end{gathered}
$$

Then $\pi_{i}=\mathbf{W}_{i} 0$.

4.2. For a principal vertex $p$ of the crystal $K$, we denote by $K_{p}$ (resp., $K^{p}$ ) the subgraph of $K$ induced by the vertex subset $\{f \in V(K): p \preceq f\}$ (resp., $\{f \in$ $V(K): f \preceq p\}$ ). The vertex $p$ is the unique minimal vertex (the source) of $K_{p}$; similarly, $p$ is the unique maximal vertex (the sink) of $K^{p}$. We shall see later that these digraphs (and their intersections) are $A_{n}$-crystals. Since

$$
K^{p}=p+K^{0} \quad K_{q}=q+K_{0}
$$

(in view of $(3.3)$ ), it suffices to consider the pre-crystals $K^{0}$ and $K_{0}$.

We will take advantages from an 'excess' description of $K_{0}$. Recall that a function $f$ on a Boolean cube is called submodular if $f(X)+f(Y) \geq f(X \cap Y)+$ 
$f(X \cup Y)$ holds for all $X, Y \subset[n]$, or, equivalently, the excesses of $f$ on all abstract tiles are nonnegative. In case of TP-functions, the latter can be weakened.

4.3. Lemma. (see [DKK-09b]). A TP-function $f$ is submodular if and only if the excesses of $f$ on all tiles of some tiling $T$ are nonnegative.

For completeness of our description, we give a short proof of this Lemma. It consists of three simple observations.

a) Every tile $R(X ; i, j)$ is contained in some tiling.

b) Any two tilings are connected be a sequence of flips.

c) If a tiling $T^{\prime}$ is obtained from a tiling $T$ by one flip, and if the excesses on all tiles of $T$ are nonnegative, then the same is true for $T^{\prime}$. (Indeed, we can reduce the zonogon to a hexagon. For a hexagon, the assertion was shown in Section 2.9.)

4.4. Theorem. A vertex (TP-function) $f$ of $K$ belongs to $K_{0}$ if and only if it is submodular.

To prove this, we first show that any vertex $f$ of $K_{0}$ is submodular. This is so for $f=0$. Therefore, it suffices to show that $\mathbf{i} f$ is submodular provided that $f$ submodular. Let $T$ be a tiling fitting to color $i$. Since $\mathbf{i}$ increases by 1 the excess on the tile $L R_{i}$ of $T$ and does not change the excesses on the other tiles of $T$, the new function if is submodular on $T$, and hence (by Lemma 4.3) it is submodular everywhere.

A proof of the converse assertion will be given in Section 6 .

4.5. Corollary. The pre-crystal $K=K[0]$ is connected.

Indeed, let $f$ be an arbitrary vertex of $K$. For a sufficiently large positive principal TP-function $p$, the function $f+p$ is submodular. By Theorem 4.4, $f+p$ is of the form $\mathbf{w} 0$ for some word $\mathbf{w}$ (composed from operators $\mathbf{i}$ ). Then $f=\mathbf{w}(-p)$. On the other hand, $p=\mathbf{v} 0$ for some word $\mathbf{v}$ (by the same theorem), whence $-p=\mathbf{v}^{-1} 0$. We obtain $f=\mathbf{w v}^{-1} 0$, and therefore the vertices $f$ and 0 are connected by edges in $K$.

There is another interesting corollary. Let $f$ be a submodular TP-function, and let its excesses on all left tiles be equal to 0 . Then $f$ is an affine function.

Note also that Theorem 4.4 implies Proposition 4.1. Indeed, for an element $p$ of the principal lattice of $K$, the following assertions are equivalent: $p$ is submodular, and $p$ is a nonnegative linear combination of the basic functions $\pi_{1}, \ldots, \pi_{n}$.

4.6. Arguing as in 3.5 , we obtain that the pre-crystal $K_{0}$ is an $A_{n}$-crystal.

According to 3.2 , a finite $A_{n}$-crystal is obtained by choosing a principal vertex $p \succeq 0$ and forming the interval in $K$ from 0 to $p$. In other words, the subgraph $K_{0}^{\bar{p}}:=K_{0} \cap K^{p}$ equipped with the induced color structure is a finite connected $A_{n}$-crystal with the source 0 and the sink $p$.

Note that the crystal $K^{0}$ is 'anti-isomorphic' to $K_{0}$. More precisely, reversing the edges of $K_{0}$ and renumbering colors $i$ as $n+1-i$, we obtain a crystal isomorphic to $K^{0}$. Such a bijection between the elements of $K_{0}$ and $K^{0}$ is called Schützenberger's map. However, it is not straightforward to give an explicit formula for this map. Next we accomplish this task for the 2-colored case. 
To this end, recall (see 2.9 or 3.5 ) that a $T P_{3}$-function with zero values on the right boundary of the hexagon $Z_{3}$ is represented by a quadruple of excesses $\left(\beta^{\prime}, \beta, \gamma^{\prime}, \gamma\right)$ satisfying two inequalities $\beta^{\prime} \leq \gamma$ and $\beta \leq \gamma^{\prime}$ together with the condition that at least one of them should be equality. It is convenient to represent this by the following crossing diagram:

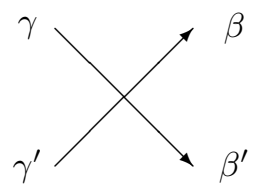

(where the arrows go from bigger values to smaller ones and their crossing symbolizes the tightness of one of the two relations). Crystal operator 1 tries to increase $\beta^{\prime}$ by one; if this is forbidden by the equality $\beta^{\prime}=\gamma$, then $\mathbf{1}$ increases $\gamma^{\prime}$. Operator $\mathbf{2}$ acts similarly in the second level.

The submodularity condition (viz. belonging to $K_{0}$ ) is given by two inequalities $\beta \geq 0$ and $\beta^{\prime} \geq 0$. Under Schützenberger's map the quadruple $\left(\beta^{\prime}, \beta, \gamma^{\prime}, \gamma\right)$ is mapped to the quadruple $\left(\widetilde{\beta}^{\prime}, \widetilde{\beta}, \widetilde{\gamma}^{\prime}, \widetilde{\gamma}\right)=\left(-\gamma,-\gamma^{\prime},-\beta,-\beta^{\prime}\right)$ represented by the diagram

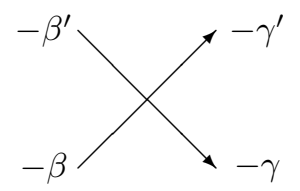

Here operator 1 on $K_{0}$ is transformed into operator $2^{-1}$ on $K^{0}$. Indeed, the action of $\mathbf{2}^{-1}$ on a quadruple $\left(\widetilde{\beta^{\prime}}, \widetilde{\beta}, \widetilde{\gamma}^{\prime}, \widetilde{\gamma}\right)$ consists in attempting to decrease $\widetilde{\gamma}$, and if this is impossible, to decrease $\widetilde{\beta}$.

4.7. Generalizing the above observation, we give an 'excess' description of vertices of $K^{p}$.

Theorem. Let $p$ be a principal TP-function in $K$. Then $f$ belongs to $K^{p}$ if and only if the inequality $\varepsilon\left(f, R R_{i}\right) \leq \varepsilon\left(p, R R_{i}\right)$ holds for each right tile $R R_{i}$.

In particular, $f$ belongs to $K^{0}$ if and only if all excesses $\varepsilon\left(f, R R_{i}\right)$ are nonpositive. The theorem can be reduced to this particular case. Indeed, $f$ belongs to $K^{p}$ $\Leftrightarrow f-p \in K^{0} \Leftrightarrow \varepsilon\left(f-p, R R_{i}\right) \leq 0 \Leftrightarrow \varepsilon\left(f, R R_{i}\right) \leq \varepsilon\left(p, R R_{i}\right)$. A proof for this particular case will be given in Section 7 .

4.8. Theorems 4.4 and 4.7 imply that a function $f \in V(K)$ belongs to the crystal $K_{0}^{p}=K_{0} \cap K^{p}$ if and only if

(a) $\varepsilon(f, R) \geq 0$ for each tile $R$;

(b) $\varepsilon\left(f, R R_{i}\right) \leq \varepsilon\left(p, R R_{i}\right)$ for $i=1, \ldots, n$.

In particular, the intersection of the crystal $K_{0}^{p}$ with the principal lattice $P$ consists of TP-functions of the form $\sum_{i} c_{i} \pi_{i}$, where $0 \leq c_{i} \leq \varepsilon\left(p, R R_{n-i+1}\right)$, yielding a rectangular integer 'box'. (Recall that $\pi_{i}$ has excess 1 at level $n-i+1$; see 3.8.) 
For a tuple $c=\left(c_{1}, \ldots, c_{n}\right)$ of nonnegative integers, we denote the crystal $K_{0}^{p}$ by $K(c)$, where $p=\sum_{i} c_{i} \pi_{i}$. Such a notation is justified by the following facts (cf. 3.3).

4.9. Proposition. (i) $c_{i}$ is the biggest number $k$ such that the function $\mathbf{i}^{k} 0$ belongs to the crystal $K(c)$.

(ii) $c_{n-i+1}$ is the biggest number $k$ such that the function $(\mathbf{i})^{-k} p$ belongs to the crystal $K(c)$.

Proof. Let us prove assertion (ii); assertion (i) follows from (ii) and general properties of $A_{n}$-crystals exposed in 3.3 (see also 7.4).

Since $p=\sum_{i} c_{i} \pi_{i}$, the excess of $p$ on any tile $R$ at height $i$ is equal to $c_{n-i+1}$ (by the definition of $\pi_{\bullet}$ in 3.8). In particular, $\varepsilon\left(p, L R_{i}\right)=c_{n-i+1}$, where, as before, $L R_{i}$ is the left tile at height $i$. Consider a tiling $T$ fitting to color $i$ (i.e. containing $\left.L R_{i}\right)$. Operator $\mathbf{i}^{-1}$ decreases the excess on $L R_{i}$ by 1 . If we apply $\mathbf{i}^{-1} k$ times, where $k \leq c_{n-i+1}$, then the excess of the function $f:=\mathbf{i}^{-k} p$ on $L R_{i}$ is still nonnegative. The excesses on the other tiles of $T$ do not change. Therefore, $f$ is submodular, and property (a) in 4.8 is satisfied. Property (b) in 4.8 is also true, due to Theorem 4.7. Hence, $f \in K(c)$. If we apply operator $\mathbf{i}^{-1}$ to $p$ more than $c_{n-i+1}$ times, then the excess on $L R_{i}$ becomes negative and the function $f$ gets outside $K(c)$.

\section{Symmetric extracts from crystals of type $A$}

5.1. Dynkin diagram $A_{n}$ has an obvious symmetry, inversion $\tau$, which transforms each color $i$ into the opposite color $n+1-i:=\bar{i}$. In a crystal $K(c)$ with source, if we change the colors of edges to the opposite ones, we again obtain an $A_{n}$-crystal, but now with the 'reversed' parameters $\bar{c}:=\left(c_{\overline{1}}, \ldots, c_{\bar{n}}\right)$.

Suppose that a tuple $c$ of parameters is symmetric, i.e., $c=\bar{c}$. Then there is a (unique) automorphism of the digraph $K(c)$ which brings each edge to an edge of the opposite color; we call it the (color-reversing) symmetry of $K(c)$ and denote by $\tau$ as well. This symmetry is extended in a natural way to paths and other objects in $K(c)$. In particular, consider a directed path $P=\left(s=v_{0}, e_{1}, v_{1}, \ldots, e_{k}, v_{k}=v\right)$ going from the source $s$ of $K(c)$ to a vertex $v$. Then there exists a (unique) path $\bar{P}$ 'symmetric' to $P$, namely, $\left(\tau\left(v_{0}\right), \tau\left(e_{1}\right), \tau\left(v_{1}\right), \ldots, \tau\left(e_{k}\right), \tau\left(v_{k}\right)\right)$; it starts at $s$ and ends at $\tau(v)$.

Let $S$ be the set of self-symmetric vertices $v$ of $K=K(c)$, i.e., $\tau(v)=v$. This set can be endowed with a structure of colored digraph, denoted as $\widetilde{K}=(S, \widetilde{E})$, with the set of colors $\widetilde{I}=I / \tau$. The construction of $\widetilde{K}$ depends on the parity of $n$ and yields either a $B_{m^{-}}$or $C_{m}$-crystal, where $m:=\lceil n / 2\rceil$.

5.2. We begin with the case of an odd $n=2 m-1$, which is slightly simpler. The factor-set $\widetilde{I}$ consists of colors $\widetilde{1}, \ldots, \widetilde{m}$. An edge of color $\widetilde{1}$ goes from a selfsymmetric vertex $u$ to a self-symmetric vertex $v$ if they are connected in $K(c)$ by a path $u \stackrel{1}{\rightarrow} w \stackrel{n}{\rightarrow} v$ (and hence, by a path $u \stackrel{n}{\rightarrow} w^{\prime} \stackrel{1}{\rightarrow} v$ ), where we write $u^{\prime} \stackrel{i}{\rightarrow} v^{\prime}$ for an edge of color $i$ from $u^{\prime}$ to $v^{\prime}$. We may write $\widetilde{\mathbf{1}}=\mathbf{1} \mathbf{n}=\mathbf{n} \mathbf{1}$. Colors $\widetilde{2}, \ldots, \widetilde{m-1}$ are defined similarly. And the edges of color $\widetilde{m}$ coincide with the original edges of color $m$ (connecting self-symmetric vertices). The resulting $m$-colored digraph $\widetilde{K}$ is called the symmetric extract from the crystal $K(c)$. 
Two simplest examples for $n=3$ (and $m=2$ ) are illustrated below. The first picture shows the $A_{3}$-crystal $K(0,1,0)$ (left) and its symmetric extract $\widetilde{K}$ (right).
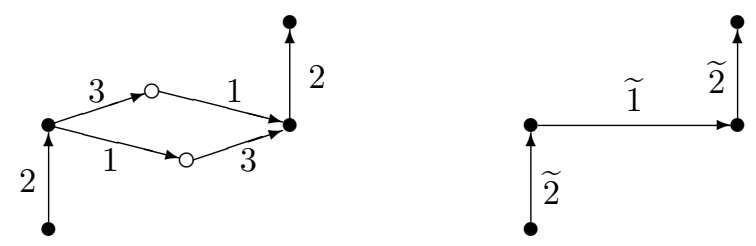

The next picture shows the $A_{3}$-crystal $K(1,0,1)$ and its symmetric extract $\widetilde{K}$.
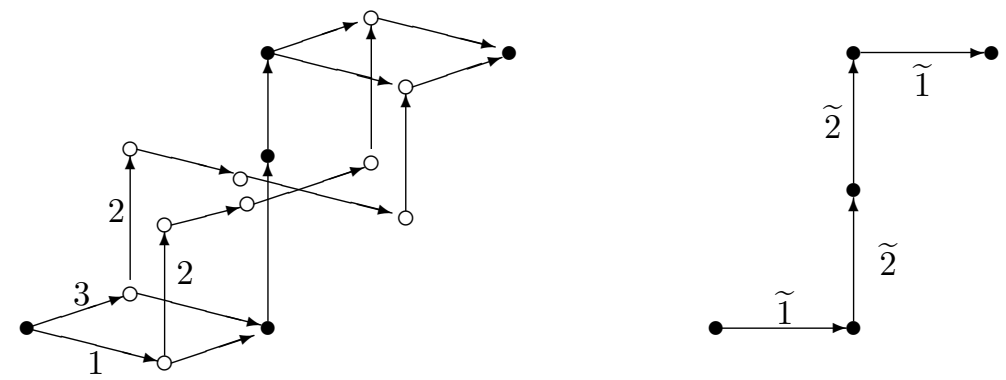

The above two extracts $\widetilde{K}$ are the simplest cases of $B_{2}$-crystals, with the parameters $(0,1)$ and $(1,0)$, called the 'fundamental' $B_{2}$-crystals (cf. [DKK-09]). Similar property takes place in a general case: for $n=2 m-1$, the symmetric extract from an $A_{n}$-crystal with a symmetric parameter $c$ is the $B_{m}$-crystal with the parameter $\left(c_{1}, \ldots, c_{m}\right)$. This property (as well as its counterpart for $A$ - and $C$-crystals in case $n=2 m$ ) follows from a general result on 'folding' on Dynkin diagrams and related crystals (see, e.g. [NS-01]); a purely combinatorial proof is given in [DKK-12]. In the rest of this section we discuss (without proofs) such an interrelation between crystals of types A,B,C in terms of our TP-model.

5.3. In case of an even $n=2 m$, the factor-set $\widehat{I}$ consists of $m$ colors $\widehat{1}, \ldots, \widehat{m}$ (here we prefer to use 'hats' rather than 'tildes'). Given a symmetric $A_{n}$-crystal $K(c)$, the edges of colors $\widehat{1}, \ldots, \widehat{m-1}$ connecting self-symmetric vertices are defined as before. For example, $\widehat{\mathbf{1}}=(\mathbf{2 m}) \mathbf{1}, \widehat{\mathbf{2}}=(\mathbf{2 m}-\mathbf{1}) \mathbf{2}$, and so on. The edges of color $\widehat{\mathbf{m}}$ are defined more tricky. Self-symmetric vertices $u, v$ are connected by edge of color $\widehat{m}$ from $u$ to $v$ if $K(c)$ contains a path of length 4 from $u$ to $v$ whose edges have colors $m, m+1, m+1, m$, in this order (implying the existence of a path with the sequence of edge colors $m+1, m, m, m+1)$. The resulting $m$-colored digraph $\widehat{K}$ is just the symmetric extract from $K(c)$.

Symmetric $A_{4}$-crystals are rather large and cumbersome to draw, and we are able to illustrate only one example here: the picture below shows the crystal $K(1,0,0,1)$ (left) and its symmetric extract (right). 

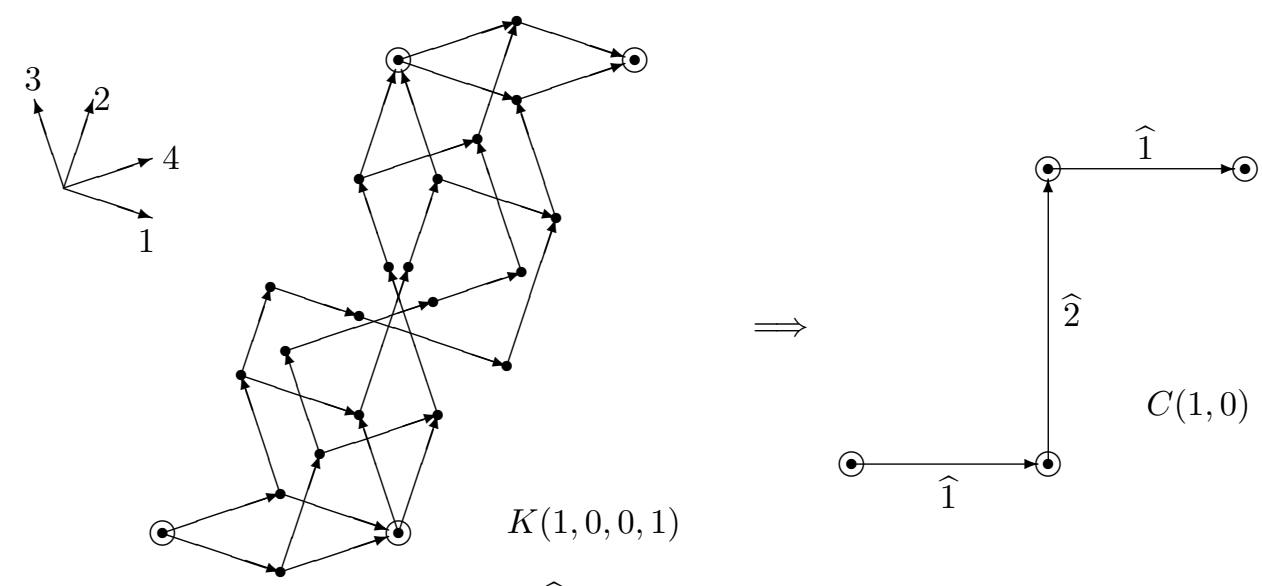

Notice that the above extract $\widehat{K}$ looks like the symmetric extract from $K(0,1,0)$ drawn in 5.2, but now the colors are interchanged. This matches the fact that a $B_{2}$ crystal with parameters $\left(c_{1}, c_{2}\right)$ is isomorphic to the $C_{2}$-crystal with the swapped parameters $\left(c_{2}, c_{1}\right)$.

5.4. Next we construct models of crystals of types $B$ and $C$ by use of symmetric TP-functions. Let us consider the inversion on the set $[n+1]$, which maps $i$ to $n+2-i$. This induces inversion $\tau$ on the Boolean cube $2^{[n+1]}$ defined by $\tau(A):=$ $\{i \in[n+1]: n+2-i \notin A\}$ for $A \subseteq[n+1]$. For example, $\tau(\emptyset)=[n+1], \tau(\{1\})=[n]$, and $\tau(\{2\})=[n+1]-\{n\}$.

One can extend $\tau$ to an inversion of the zonogon $Z=Z_{n+1}$. To this end, we make the mirror reflection w.r.t. the middle horizontal line in $Z$. This maps $Z$ onto itself (interchanging the top and bottom vertices), and a point $v$ of the zonogon represented by a set $A \subseteq[n+1]$ is mapped to the point $\tau(v)$ represented by $\tau(A)$.

For a function $f$ on $2^{[n+1]}$, we can consider the symmetric function $\tau^{*}(f)$ defined by $\tau^{*}(f)(A):=f(\tau(A))$. Let $\widetilde{T P}$ denote the set of (self)-symmetric TP-functions $f$ on $2^{[n+1]}$, i.e., $\tau^{*}(f)=f$. According to 5.2 and 5.3 , this set can be endowed by the structure of $B$ - or $C$-crystal. We will describe the action of crystal operators on the set $\widetilde{T P}$. This depends on the parity of $n$.

5.5. The case of an odd $n=2 m-1$ is simpler because in this case there exist symmetric tilings. Below we draw two symmetric tilings for $n=3$.
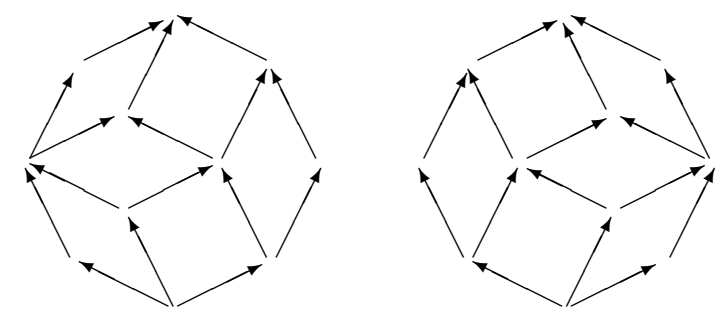

If a tiling $T$ is symmetric (i.e. $\tau(T)=T$ ), then any symmetric function on the set of vertices of $T$ can be uniquely extended to a symmetric TP-function on $2^{[n+1]}$. To define operators $\widetilde{\mathbf{i}}$, take a symmetric tiling $T$ fitting to color $i$ (which fits to color $\tau(i)=n+1-i$ as well). (Arguing as in 3.3, one can show that such a tiling does 
exist.) When $i \neq m$, we simultaneously increase by 1 the values at the symmetric vertices $[i]$ and $\tau([i])=[2 m-i]$, preserving the values at the other vertices of $T$. When $i=m$, we increase by 1 the value at the self-symmetric point $[m]$.

5.6. For an even $n=2 m$, there are no symmetric rhombus tilings. For this reason, we work with symmetric hexagonal-rhombus tilings. 'Tiles' of such a tiling $T$ are either usual tiles $R$ (then $T$ should contain their symmetric tiles $\tau(R)$ ) or symmetric (relative to $\tau$ ) hexagons. As before, any symmetric TP-function is determined by its values at the vertices of such a $T$. To show this, it suffices to consider in detail the case of a single hexagon, i.e., the case $n=2$. (One can understand this case as taking the symmetric extract from an $A_{2}$-crystal.)

The picture below illustrates the hexagon $Z_{3}$ with two possible rhombus tilings on it, and indicates the values of a symmetric TP-function.

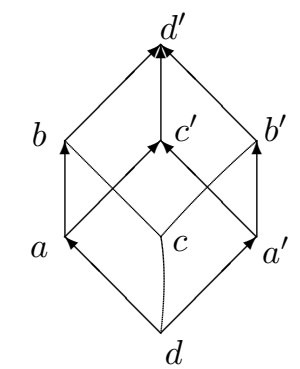

By the symmetry, we have obvious equalities $a=b, a^{\prime}=b^{\prime}, c=c^{\prime}$, and $d=d^{\prime}$. The $T P_{3}$-condition takes the form

$$
2 c=\max \left(a+b^{\prime}, b+a^{\prime}\right)=a+a^{\prime} .
$$

We observe that the values of a symmetric TP-function on the boundary vertices determine its values at both inner vertices by the rule: $c=\left(a+a^{\prime}\right) / 2$.

One can see how the operator $\mathbf{1 2 2 1}=\mathbf{2 1 1 2}$ acts on a symmetric TP-function. Namely, $\mathbf{1}$ increases (by 1) $a$ and $c^{\prime}$, then $\mathbf{2}$ increases $b$, then the second application of $\mathbf{2}$ increases $b$ and $c$, and the last application of $\mathbf{1}$ increases $a$. As a result, $a$ and $b$ increase by 2 (while $c$ and $c^{\prime}$ increase by 1 ).

Returning to an arbitrary even $n$, we have the following description of crystal operators $\widehat{\mathbf{1}}, \ldots, \widehat{\mathbf{m}}$. For $i<m$, operator $\widehat{\mathbf{i}}$ acts as follows. Take a symmetric hexagonal-rhombus tiling fitting to color $i$ (and $2 m+1-i$ ) and increase by 1 the values of the function at the vertices $[i]$ and $[2 m+1-i]$. As to the action of $\widehat{\mathbf{m}}$, one should take a symmetric hexagonal-rhombus tiling containing the hexagon using the vertices $[m-1],[m],[m+1],[m+2]$, and increase by 2 the values of the function at $[m]$ and $[m+1]$ (preserving the values at the other vertices of the tiling). Two symmetric hexagonal-rhombus tilings for $n=4$ are drawn in the picture.
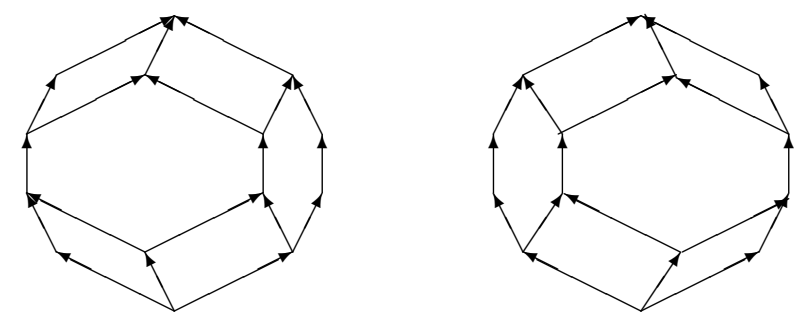
In fact, we have described above the free TP-crystals of types $B$ and $C$. To obtain crystals $\widetilde{K}(\widetilde{c})$ (with sources), one should extend $\widetilde{c}$ to an appropriate symmetric tuple $c$ and consider symmetric TP-functions in the crystal $K(c)$ (we omit details).

\section{Proof of Theorem 4.4}

We have already shown that if a TP-function $f$ is submodular, then it continues to be submodular after application of every crystal operator $i$. It remains to show the following 'converse' property: if $f$ is a nonzero submodular integer TP-function, then for some color $i$, the function $\mathbf{i}^{-1} f$ is submodular as well.

An idea of the proof is simple. Due to the submodularity, the excess of $f$ on any tile is nonnegative. Hence, there exists a tile $R$ with a strictly positive excess. The idea consists in showing the existence of a 'more left' tile also having positive excess. Eventually we obtain a 'leftmost' tile with positive excess which is pressed to the left boundary of the zonogon $Z$, i.e., the left tile $L R_{i}$. Then we can apply to $f$ the operator $\mathbf{i}^{-1}$ and the obtained function $\mathbf{i}^{-1} f$ is again submodular, whence the result easily follows.

First of all we should explain which tile is meant to be 'more left' to a given tile.

Assign to each tile $R=(A ; i, j)$ the following permutation $\sigma(R)$ on $[n+1]$. (Recall that $A \subset[n+1], i, j \notin A$ and $i<j$.) We set $\sigma(R):=(A, i, j, B)$, where $B:=[n+1]-A i j$, and the sets $A$ and $B$ are ordered by increasing their elements. For instance, if $a$ is the biggest element of $A$, and $b$ is the smallest element of $B$, then the permutation has the form ...aijb.... As is explained in Section 2, there exists a tiling containing both the tile $R$ and the snake $\sigma(R)$. If $R$ is the left tile, then $\sigma(R)$ is the identical permutation $i d$ (corresponding to $l b d(Z)$ ). We say that a tile $R^{\prime}$ is more left than a tile $R$ if $\sigma\left(R^{\prime}\right)$ is closer to $i d$ than $\sigma(R)$ (in the sense of weak Bruhat order).

Now suppose that $R$ is a non-left tile with positive excess. Let $\sigma(R)=$ $(A, i, j, B)$. Let $a$ be the biggest element of $A$, and $b$ the smallest element of $B$. We consider two cases.

Case 1: $a>j$. Extend $R$ to the hexagon with the bottom vertex $A-a$ and the left boundary going along edges of colors $i, j, a$ (in this order). See the picture:

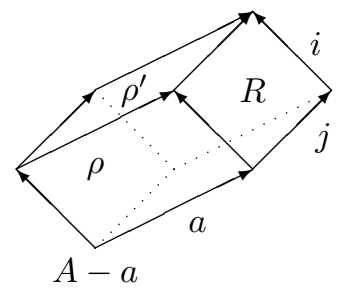

By a remark in 2.9, at least one of the left tiles in the hexagon, $\rho$ or $\rho^{\prime}$, has positive excess. The permutation $\sigma\left(\rho^{\prime}\right)$ has the form $((A-a) \cup i, j, a, B)$ and is closer to $i d$ than $\sigma(R)=(A, i, j, B)$. And the permutation $\sigma(\rho)$, which has the form $(A-a, i, j, B \cup a)$, also is closer to $i d$ than $\sigma(R)$.

Case 2: $i<a<j$. Extend $R$ to the hexagon with the bottom vertex $A-a$ and the left boundary going along edges of colors $i, a, j$. See the picture: 


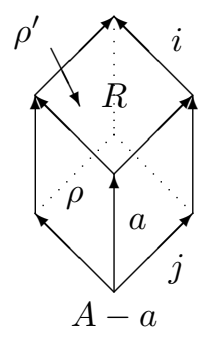

As before, at least one of the left tiles in the hexagon has positive excess. In all cases the corresponding new permutation is closer to $i d$ that $\sigma(R)$.

Arguing similarly for $B$, we obtain that if $b<j$, then there exists a more left tile with positive excess than $R$.

Finally, assume that $R$ is such that $a<i$ and $b>j$. This implies that $j=i+1$ and the tile $R$ is pressed to $l b d(Z)$.

Theorem 4.4 is proven.

\section{Proof of Theorem 4.7}

7.1. In Section 4 we introduced the important subgraph $K^{0}$ in $K$. Theorem 4.7 gives another description of this subgraph. More precisely, let $K_{-}$be (the subgraph induced by) the set of functions $f$ in $K$ which have nonpositive excesses on all right tiles: $\varepsilon\left(f, R R_{i}\right) \leq 0$ for any $i=1, \ldots, n$. Theorem 4.7 says that $K^{0}=K_{-}$.

To prove this, we have to show two properties. The first one (see parts 7.2-7.3 below) is that the set $K_{-}$is stable under action of operators $\mathbf{i}^{-1}$. Together with the trivial fact that $0 \in K_{-}$, this gives the inclusion $K^{0} \subseteq K_{-}$. The second one is that 0 is the (unique) sink of the digraph $K_{-}$. In other words, we have to prove that $\mathbf{i} 0 \notin K_{-}$(this is rather easy and is shown in 7.4), and that if for a function $f \in K_{-}$, the function $\mathbf{i} f$ do not belong to $K_{-}$for any $i$, then $f=0$ (this is more difficult and is shown in 7.5-7.7).

7.2. For $i \in[n]$, we write $\bar{i}$ for $n+1-i$. We assert that if $j \neq \bar{i}$, then the excess on the tile $R R_{j}$ does not change under the action of operator $\mathbf{i}^{-1}: \varepsilon\left(\mathbf{i}^{-1} f, R R_{j}\right)=$ $\varepsilon\left(f, R R_{j}\right)$. This follows from the observation that in case $i+j \neq n+1$, there exists a tiling containing both tiles $L R_{i}$ and $R R_{j}$. It is easy to construct explicitly such a tiling, and we leave it to the reader.

7.3. The case $j=\bar{i}=n-i+1$ is less trivial. The above argument does not work because the corresponding tiling does not exist. Also in a general case operator $\mathbf{i}^{-1}$ may change the excess on the tile $R R_{j}$. We assert that this excess either preserves or decreases by 1.

To prove this, we draw the 'track' of color $i+1$ pressed to a lower boundary of the zonogon as shown in the picture: 


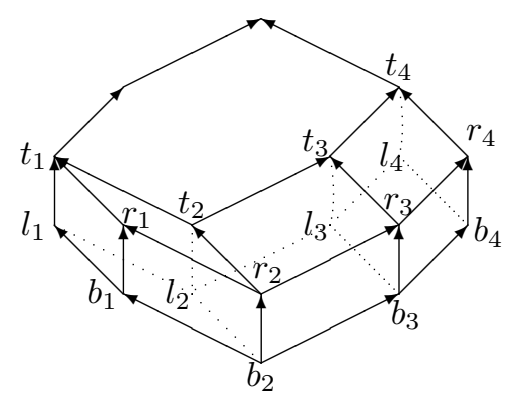

When this track is removed, in the rest of the zonogon we can draw a similar track of color $i$ pressed to a lower boundary. These two tracks form a partial tiling 'beginning' with the left tile $L R_{i}$, which we call the 'visible' tiling. Also within the same domain we can constract another tiling; it is formed by a track of color $i$ and a track of color $i+1$. This 'hidden' tiling, drawn by dotted lines in the above picture, 'ends' with the right tile $R R_{j}$.

By combining both tilings, we can see a gallery of cubes whose facets include tiles with edges of colors $i, i+1$. We label the vertices by letters $b, l, t, r$ with corresponding indices as indicated in the above picture. When we apply operator $\mathbf{i}^{-1}$ to a function $f$, the value at the vertex $l_{1}$ decreases by 1 whereas the values at the vertices $b_{\bullet}, r_{\bullet}$ and $t_{\bullet}$ do not change. We are going to compute the change of the value at the vertex $l_{n}$ (and simultaneously at all $l_{1}, \ldots, l_{n}$ ). We will prove in 7.5 a more general fact: for any $k$, the value at the point $l_{k}$ either does not change or decreases by 1 .

7.4. For simplicity let us first consider the case of zero function $f$. We assert that the function $g=\mathbf{i}^{-1} 0$ takes value -1 at all points $l_{1}, \ldots, l_{n}$. Indeed, by the TPrelation applied to first cube, and the equality $g\left(l_{1}\right)=-1$, we obtain $g\left(l_{2}\right)=-1$. From the latter, we obtain $g\left(l_{3}\right)=-1$, and so on until we get $g\left(l_{n}\right)=-1$.

The same argument shows that for any integer $m$, the function $\mathbf{i}^{m} 0$ takes value $m$ at the point $l_{n}$. In particular, the excess of the function on the right tile $R R_{\bar{i}}$ is $m$. Together with the assertion in 7.2, this proves the first assertion in Proposition 4.9.

7.5. Let us return to a general TP-function $f$ and to the assertion in 7.3: the application to $f$ of operator $\mathbf{i}^{-1}$ either does not change the value at the point $l_{k}$ or decreases it by 1 . We prove this by induction on $k$; for $k=1$ the assertion is trivial. Let us consider $k$-th cube in the above picture; it is as illustrated:

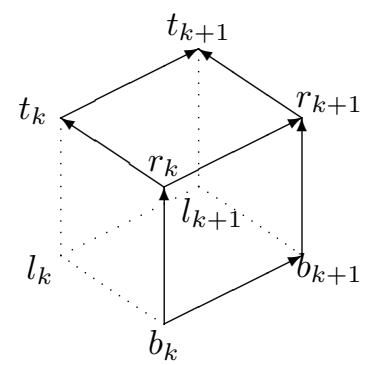

Due to TP-relation (2.1), we have the equality

$$
f\left(r_{k}\right)+f\left(l_{k+1}\right)=\max \left(f\left(t_{k}\right)+f\left(b_{k+1}\right), f\left(l_{k}\right)+f\left(r_{k+1}\right)\right) .
$$


By induction the value at $l_{k}$ either does not change or decreases by 1 . Also the values at the points $b, t, r$ do not change. If the value at $l_{k}$ does not change, then the value at $l_{k+1}$ does not change as well. If the value at $l_{k}$ decreases by 1 , then the value at $l_{k+1}$ either does not change or decreases by 1 .

7.6. Proposition. Suppose that a function $f$ in $K_{-}$has the following property: if does not belong to $K_{-}$for any $i=1, \ldots, n$. Then $f=0$.

To show this, it is convenient to work with the standard tiling. It has the track of color 1 pressed to the left boundary of zonogon. Denote the vertices of this track (or $l b d(Z))$ as $\emptyset=t_{0}, t_{1}, \ldots, t_{n}, t_{n+1}$, and the vertices of the right boundary of the track as $r_{0}=\emptyset, r_{1}, \ldots, r_{n}$. On the other hand, the vertices $r_{j}$ belong to the left boundary of the smaller zonogon $Z^{\prime}$ obtained by removing from $Z$ the track of color 1 . Then $Z^{\prime}$ is just the zonogon induced by color $2, \ldots, n+1$ (or vectors $\left.\xi_{2}, \ldots, \xi_{n+1}\right)$.

First we consider the actions of operators $\mathbf{i}$ for $i=2, \ldots, n$. These actions are 'not visible' on the standard tiling because it does not fit to any of these colors. To make them 'visible', we extend each pair of tiles of types $1 i$ and $1(i+1)$ to the hexagon as illustrated in the picture:

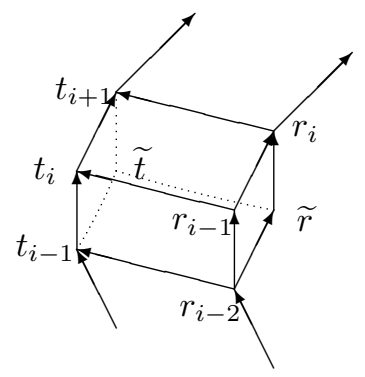

In addition, we consider the 'dotted tiling' on the hexagon; its interior contains vertex $\widetilde{t}$. By TP-relation (2.1), we have

$$
f(\widetilde{t})+f\left(r_{i-1}\right)=\max \left(f\left(t_{i-1}\right)+f\left(r_{i}\right), f\left(t_{i}\right)+f(\widetilde{r})\right)
$$

and a similar relation holds for the function $g:=\mathbf{i} f$.

Recall that operator $\mathbf{i}$ increases by 1 the value at $t_{i}$ and does not change values at $t_{i-1}, t_{i+1}, r_{i}, r_{i-2}$, and $\widetilde{r}$. The above-mentioned TP-relations give two possible cases:

1) either $f\left(t_{i-1}\right)+f\left(r_{i}\right)>f\left(t_{i}\right)+f(\widetilde{r})$, and therefore the value at $r_{i-1}$ does not change (i.e., $\left.f\left(r_{i-1}\right)=g\left(r_{i-1}\right)\right)$; by 1 .

2) or $f\left(t_{i-1}\right)+f\left(r_{i}\right) \leq f\left(t_{i}\right)+f(\widetilde{r})$, and therefore the value at $r_{i-1}$ increases

7.7. We assert that only the second case is realizable. Indeed, suppose that the value at $r_{i-1}$ does not change. Then operator $\mathbf{i}$ does not change the function $f$ within the zonogon $Z^{\prime}$. More precisely, for a tiling $T^{\prime}$ on $Z^{\prime}$ fitting to color $i$, the values at vertices of $T^{\prime}$ preserve. Then the values at vertices of any tiling on $Z^{\prime}$ are not changed, in particular, at the vertices of the right tiles $R R_{j}, j \neq n+1$. Therefore, the excesses on these tiles continue to be nonpositive. The excess on 
$R R_{n}$ is not changed as well (see 7.3). But this means that the function if belongs to $K_{-}$, contrary to the supposition.

Thus, we have

$$
f\left(t_{i-1}\right)+f\left(r_{i}\right) \leq f\left(t_{i}\right)+f(\widetilde{r})
$$

and the action of $\mathbf{i}$ increases by one the value at the point $r_{i-1}$. Hence, in the restriction of $f$ to the Boolean cube $2^{\{2, \ldots, n+1\}}$ operator $\mathbf{i}$ acts precisely as the corresponding operator of the same color $i(i=2, \ldots, n)$. By the inductive assumption, we obtain that the function $f$ is zero on the restriction to $2^{\{2, \ldots, n+1\}}$.

In particular, $f\left(r_{i}\right)=f(\widetilde{r})=0$. Therefore, relations (7.1) give

$$
f\left(t_{1}\right) \leq f\left(t_{2}\right) \leq \ldots \leq f\left(t_{n}\right) .
$$

7.8. Finally, we show that all values $f\left(t_{i}\right)$ are zeros (implying that $f$ is zero on all vertices of the standard tiling, whence it is zero everywhere). Here we use operator 1 (which was not used so far).

Let us consider again the standard tiling and the tracks of colors 1 and 2 in it. They cover some 'horn' domain; see the following picture (where vertices on the tracks are labeled as $\left.t_{\bullet}, r_{\bullet}, b_{\bullet}\right)$.

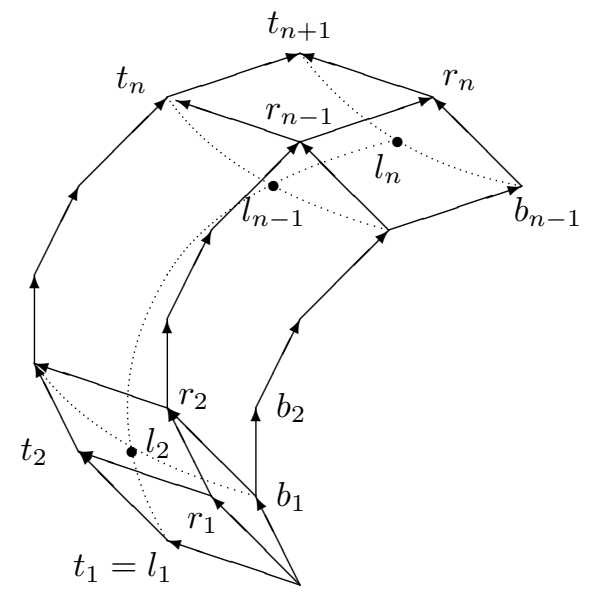

As before, we also consider another, 'hidden' tiling on the same 'horn' domain; new vertices are labeled as $l_{\bullet}$. Note that the values of $f$ at the vertices $b_{\bullet}$ and $r_{\bullet}$ are equal to 0 because they belong to the smaller Boolean cube. The values of $\mathbf{1} f$ are equal to 0 at these points as well, because the standard tiling fits to color 1 . Due to TP-relations, we have

$$
f\left(r_{i-1}\right)+f\left(l_{i}\right)=\max \left(f\left(t_{i-1}\right)+f\left(r_{i}\right), f\left(t_{i}\right)+f\left(b_{i-1}\right)\right) .
$$

Since $f$ is zero at $b_{\bullet}$ and $r_{\bullet}$, these relations give

$$
f\left(l_{i}\right)=\max \left(f\left(t_{i-1}\right), f\left(t_{i}\right)\right) .
$$

Similarly,

$$
(\mathbf{1} f)\left(l_{i}\right)=\max \left((\mathbf{1} f)\left(t_{i-1}\right),(\mathbf{1} f)\left(t_{i}\right)\right) .
$$

We are interested in the value at the point $l_{n}$ because it is equal to the excess on the right tile $R R_{n}$. We know that $(\mathbf{1} f)\left(t_{i}\right)=f\left(t_{i}\right)$ for $i=2, \ldots, n$, and that 
$(\mathbf{1} f)\left(t_{1}\right)=f\left(t_{1}\right)+1$. Moreover, in view of (7.2), we have

$$
f\left(l_{n}\right)=f\left(t_{n}\right) \text { and } \mathbf{1} f\left(l_{n}\right)=\max \left(f\left(t_{1}\right)+1, f\left(t_{n}\right)\right) .
$$

Now since $f \in K_{-}$, we have $f\left(l_{n}\right) \leq 0$. And $\mathbf{1} f \notin K_{-}$implies $\mathbf{1} f\left(l_{n}\right)>0$. It is possible only if $f\left(t_{n}\right) \leq 0$ and $f\left(t_{1}\right) \geq 0$. Together with $(7.2)$, this gives $f\left(t_{i}\right)=0$ for each $i=1, \ldots, n$.

This completes the proof of the theorem.

\section{References}

[DKK-07] V.I. Danilov, A.V. Karzanov, and G.A. Koshevoy, Combinatorics of regular $A_{2}$-crystals. J. of Algebra 310 (2007) 218-234.

[DKK-08] V.I. Danilov, A.V. Karzanov, and G.A. Koshevoy, The crossing model for regular $A_{n^{-}}$ crystals. J. of Algebra 320 (2008) 3398-3424.

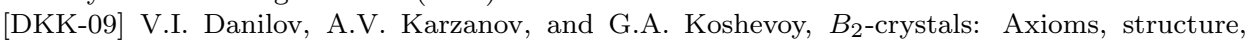
models. J. Combin. Theory, Ser. A 116 (2009) 265-289.

[DKK-09b] V.I. Danilov, A.V. Karzanov, and G.A. Koshevoy, Tropical Plücker functions and their bases, in: Tropical and Idempotent Mathematics (ed. G.L. Litvinov and S.N. Sergeev), Contemporary Math. 495 (2009) 127-158.

[DKK-10] V.I. Danilov, A.V. Karzanov, and G.A. Koshevoy, Plücker environments, wiring and tiling diagrams, and weakly separated set-systems. Adv. Math. 224 (2010) 1-44.

[DKK-10b] V.I. Danilov, A.V. Karzanov, and G.A. Koshevoy, Systems of separated sets and their geometrical models. Uspekhi Matem. Nauk 65 No. 4 (2010) 132-217 (in Russian). English translation in Russian Math. Surveya 65:4 (2010) 659-740.

[DKK-12] V.I. Danilov, A.V. Karzanov, and G.A. Koshevoy, On the combinatorial structure of crystals of types $A, B, C$. ArXiv: 1201.4549 [math.CO], 2012.

[El-97] S. Elnitsky, Rhombic tilings of polygons and classes of reduced words in Coxeter groups. J. Combin. Theory, Ser. A 357 (1997) 193-221.

[HS-07] A. Henriques and D. Speyer, The multidimensional cube recurrence, ArXiv:0708.2478v1[math.CO], 2007.

[Kam-10] J. Kamnitzer, Mirković-Vilonen cycles and polytopes. Annals of Math. 171 (2010), $731-777$.

[NS-01] S.Naito and D.Sagaki, Lakshmibai-Seshadri paths fixed by a diagram authomorphism. J. of Algebra 245 (2001) 395-412.

[KN-94] M. Kashiwara and T. Nakashima, Crystal graphs for representations of the q-analogue of classical Lie algebras, J. of Algebra 165 (1994) 295-345.

[Lu-93] G. Lusztig, Introduction to quantum groups. Progress in Mathematics 110, Birkhauser, 1993.

[Lit-95] P. Littelmann, Paths and root operators in representation theory. Annals of Math. 142 (1995) 499-525.

[Ste-03] J.R. Stembridge, A local characterization of simply-laced crystals. Transactions of the Amer. Math. Soc. 355 (12) (2003) 4807-4823.

Central Institute of Economics and Mathematics of the RAS, 47, Nakhimovskil Prospect, 117418 Moscow, Russia (V.I. Danilov); Inst. for System Analysis of the RAS, 9, Prosp. 60 Let Oktyabrya, 117312 Moscow, Russia (A.V. Karzanov); Central Institute of Economics and Mathematics of the RAS and Laboratoire J.-V.Poncelet, 11, Bolshoy Vlasyevskit Pereulok, 119002 Moscow, Russia (G.A. Koshevoy). 